\title{
Combination of High- and Low-Fluenced Erbium:Yttrium- Aluminum-Garnet Laser Treatments for Striae Distensae in Asian Patients
}

\author{
Heesu Kim ${ }^{1}$ \\ Zhenlong Zheng ${ }^{1,2}$ \\ Sung Bin $\mathrm{Cho}^{1,3}$
}

\author{
${ }^{1}$ Department of Dermatology and Cutaneous \\ Biology Research Center, International St. Mary's \\ Hospital, Catholic Kwandong University College of \\ Medicine, Incheon, Korea \\ ${ }^{2}$ Department of Dermatology, Yanbian University \\ Hospital, Yanji, China \\ ${ }^{3}$ Kangskin Dermatology Clinic, Seoul, Korea
}

\begin{abstract}
The safety and efficacy of 2,940-nm erbium (Er):yttrium-aluminumgarnet (YAG) laser treatments have been remarkably improved by incorporating technology in which high-fluenced, fractionated laser pulses are delivered to target areas, leaving the surrounding skin uninjured, and low-fluenced resurfacing technology delicately generates photothermal tissue reactions in the uppermost epidermis. In this report, we describe two Korean patients with striae distensae on the lower back and abdomen, respectively, who were effectively treated with a single session of combined high-fluenced, fractionated Er:YAG laser and lowfluenced Er:YAG resurfacing laser treatment. High-fluenced, 2,940-nm fractionated Er:YAG laser treatment was delivered along the striae distensae lesions at a fluence of $37 \mathrm{~J} / \mathrm{cm}^{2}$ and coverage of $11 \%$ over a single pass. Immediately after high-fluenced, fractionated Er:YAG laser treatment, low-fluenced Er:YAG laser resurfacing was performed with a beam size of $4 \mathrm{~mm}$ and a fluence of $2.0 \mathrm{~J} / \mathrm{cm}^{2}$ at the expected penetration depth of $8 \mu \mathrm{m}$ and an overlap of $30 \%$ over a single pass. Two to three months after treatment, both patients exhibited marked improvements in the striae distensae lesions without remarkable major side effects. Our data demonstrate that combined high-fluenced, fractionated Er:YAG laser and low-fluenced Er:YAG resurfacing laser treatment can be used to effectively treat striae distensae in Asian patients.
\end{abstract}

\section{Key words}

Erbium:yttrium-aluminum-garnet laser; Fractionated laser; Intraepidermal laser resurfacing; Stria distensa; Stria alba
Received September 18, 2018

Accepted October 14, 2018

\footnotetext{
Correspondence

Sung Bin Cho

Department of Dermatology and Cutaneous

Biology Research Center, International St. Mary's Hospital, Catholic Kwandong University College of Medicine, 25 Simgok-ro, Seo-gu, Incheon 22711, Korea

Tel.: +82-32-290-3141

Fax: $+82-32-290-3142$

E-mail: drsbchođagmail.com

(C) Korean Society for Laser Medicine and Surgery

(c) This is an open access article distributed under the terms of the Creative Commons Attribution NonCommercial License (http://creativecommons.org/ licenses/by-nc/4.0) which permits unrestricted noncommercial use, distribution, and reproduction in any medium, provided the original work is properly cited.
} 


\section{INTRODUCTION}

Striae distensae begin as pink or red flat lesions that histopathologically exhibit inflammatory cell infiltration with mast cell degranulation and elastolysis (striae rubrae). ${ }^{1,2}$ Striae distensae progress to hypopigmented and atrophic lesions exhibiting scar-like histopathological features, including thinning of the epidermis, thin collagen bundles in parallel arrangements to the flattened epidermis, and loss of collagen and elastin (striae albae).1,2 Striae rubrae have been treated with topical retinoids, pulsed dye laser, copper bromide laser, intense pulsed dye laser, and superficial mechanical dermabrasion. ${ }^{1-3}$ Meanwhile, striae albae have been treated with mid to deep mechanical dermabrasion, 308-nm excimer laser, fractional laser treatment, radiofrequency treatment, autologous platelet-rich plasma injection treatment, and transcutaneous pneumatic injection of $20 \%$ glucose solution or tissue activators. ${ }^{2,45}$

Ablative 2,940-nm erbium (Er):yttrium-aluminumgarnet (YAG) lasers achieve satisfactory therapeutic outcomes in treatment of various photodamaged or atrophic skin disorders. ${ }^{4,6}$ However, high incidences of immediate post-Er:YAG bleeding and oozing, as well as prolonged post-treatment erythema and dyschromia, limit the use thereof in Asian patients. Nonetheless, notable improvements in the efficacy and safety of Er:YAG laser treatment have been attained by incorporating fractional technology therein to deliver high-fluenced laser pulses to target areas, leaving the surrounding skin uninjured, and by adopting low-fluenced Er:YAG resurfacing technology that generates photothermal tissue reactions in the uppermost epidermis, with coverage percentage over $100 \% .^{7-9}$ Herein, we describe two Korean patients with striae distensae on the lower back and abdomen, respectively, who were effectively treated with a single session of combined highfluenced, fractionated Er:YAG laser and low-fluenced Er:YAG resurfacing laser treatment.

\section{CASE REPORT}

Two Korean patients (33-year-old and 29-year-old females) visited our clinic presenting with multiple, linear, atrophic skin lesions on the trunk. The former patient presented with hypopigmented and atrophic lesions that had persisted over 12 years (Fig. 1A), while the latter patient exhibited erythematous and atrophic lesions that had progressed over 6 months (Fig. 2A). Neither of the patients reported treatment history for atrophic skin lesions, including systemic or topical retinoid therapy, pulsed dye laser, fractional laser treatment, radiofrequency treatment, thread implantation, and injection therapies with fillers or tissue activators, or pertinent medical or family history. The patients were clinically diagnosed with striae distensae, and were scheduled to undergo combined high- and low-fluenced Er:YAG laser treatment.

After obtaining written informed consent, the patients' skin was cleansed with $70 \%$ ethanol, and topical anesthetic cream leutectic mixture of $2.5 \%$ lidocaine $\mathrm{HCl}$ and $2.5 \%$ prilocaine, EMLA; Astra Pharmaceuticals, Westborough, MA, USA) was applied for 1 hour. Then, highfluenced, 2,940-nm fractionated Er:YAG laser treatment (Sciton Joule; Sciton, Palo Alto, CA, USA) using a computerized scanner (Sciton) was delivered along the striae

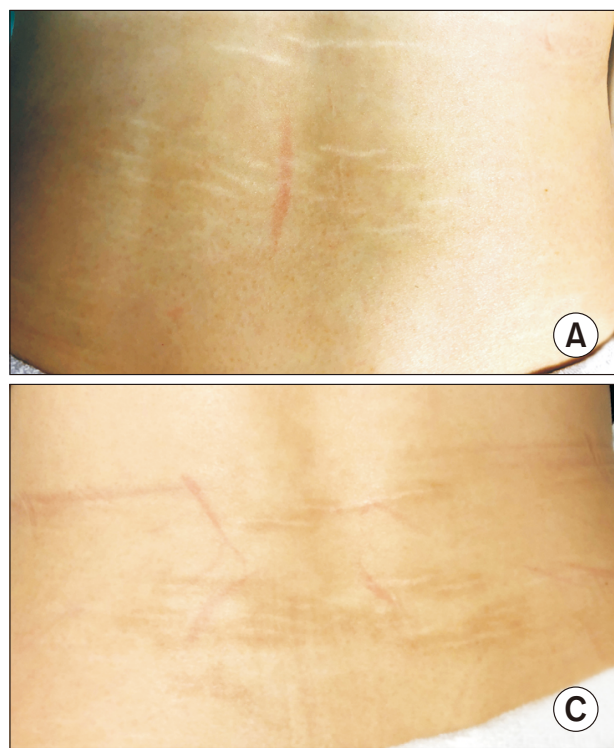

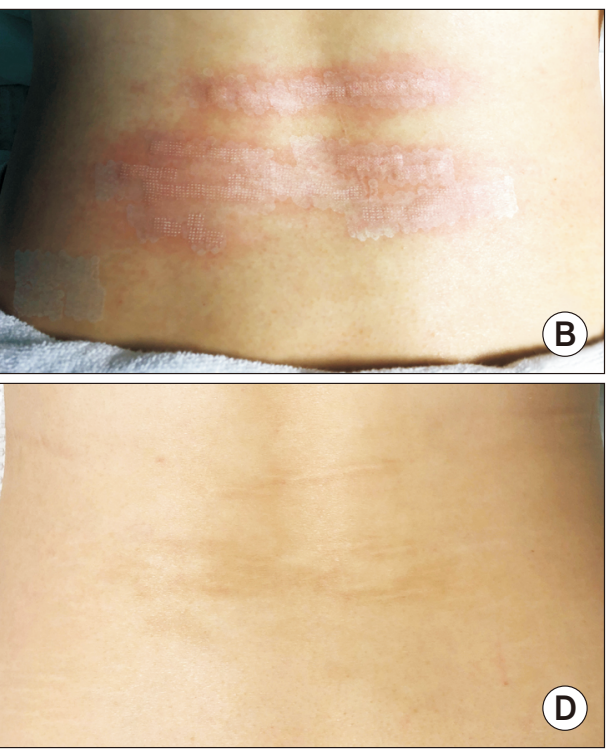

Fig. 1. Photographs of a 33-year-old female patient with striae distensae on the lower back. (A) Baseline and (B) immediately, (C) one month, and (D) three months after one session of combined high- and lowfluenced erbium (Er):yttrium-aluminum-garnet (YAG) laser treatment. 


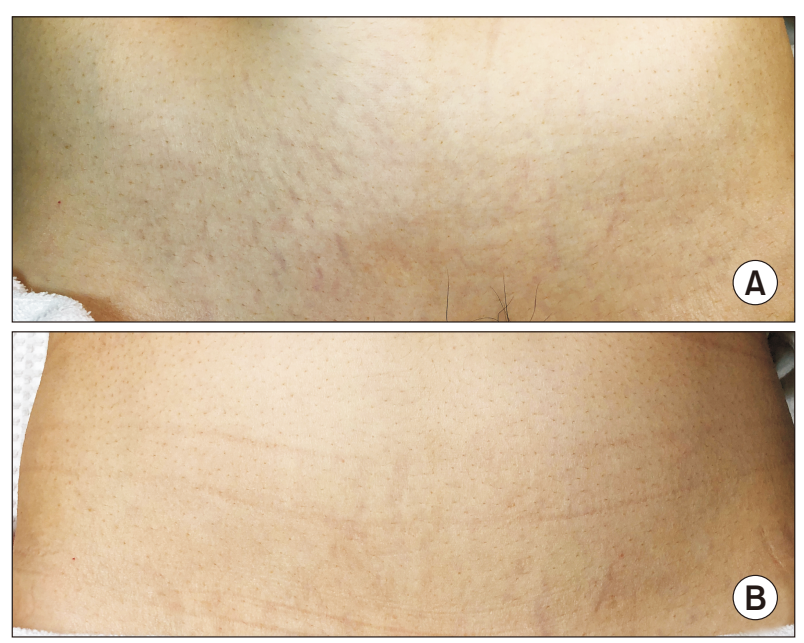

Fig. 2. Photographs of a 29 -year-old female patient with striae distensae on the abdomen. (A) Baseline and (B) two months after one session of combined high-and low-fluenced Er:YAG laser treatment.

distensae lesions. The treatment settings comprised a fluence of $37 \mathrm{~J} / \mathrm{cm}^{2}$ at the expected penetration depth of $150 \mu \mathrm{m}$, without an additional coagulation setting, and a percent coverage of $11 \%\left(60\right.$ spots $\left./ \mathrm{cm}^{2}\right)$ over a single pass. Each pulse elicited a rectangular ablative pattern of high-fluenced fractionated Er:YAG laser-induced thermal injury that was generated by 25 microbeams uniformly arranged as five spots $\times$ five spots $(5 \mathrm{~mm} \times 5 \mathrm{~mm})$. Postfractionated Er:YAG laser pinpoint bleeding was well controlled by compression with dry gauze.

Immediately after high-fluenced fractionated Er:YAG laser treatment, low-fluenced Er:YAG laser resurfacing (Sciton Joule; Sciton) was performed with a beam size of $4 \mathrm{~mm}$ and a fluence of $2.0 \mathrm{~J} / \mathrm{cm}^{2}$ at the expected penetration depth of $8 \mu \mathrm{m}$ and an overlap percentage of $30 \%$ over a single pass using a computerized scanner (Sciton). Each treatment was delivered to the striae distensae lesions by generating a rectangular ablative pattern composed of nine spots uniformly arranged as three spots $\times$ three spots $(9 \mathrm{~mm} \times 9 \mathrm{~mm})$ at the overlap percentage of $30 \%$. The treatment endpoint was a mild to moderate frosted appearance immediately after low-fluenced Er:YAG laser treatment (Fig. 1B). After combination treatment, the treated areas were cooled with icepacks. No prophylactic systemic corticosteroids or antibiotics were prescribed. The patients were recommended to avoid sun exposure and to use emollients. The use of topical bleaching and retinoid agents during the follow-up period was not recommended.

Pain during the treatment was tolerable with the use of topical anesthetic cream. Immediately after combined high- and low-fluenced Er:YAG laser treatment, both patients exhibited erythematous swelling on the treated areas with mild oozing that spontaneously disappeared within 12 hours. Pinpoint crusts and filmy scales were found along the striae lesions at post-treatment day 1. Most of the post-Er:YAG crusts and scales fell off spontaneously over 5-7 days. Two to three months after the treatment, both patients exhibited marked improvements in the striae distensae lesions (Fig. 1C, 1D, and 2B). One patient, however, experienced postinflammatory hyperpigmentation in the treated areas on the lower back that gradually disappeared within 3 months. Otherwise, no remarkable major side effects, including petechiae or bruising, excessive bleeding, burn, secondary viral or bacterial infection, prolonged edema or erythema, post-therapy hypopigmentation, atrophic or hypertrophic scarring, contracture, and keloid formation, were encountered.

\section{DISCUSSION}

Treatment modalities for striae distensae using energydelivering devices include nonablative or ablative fractional laser treatment and non-invasive or invasive radiofrequency treatment. ${ }^{4,5,10}$ Ablative fractional laser treatment and invasive radiofrequency treatment have been shown to induce stratifin and transforming growth factor- $\beta 1$ to improve scar-like histopathologic features in striae distensae. ${ }^{4,5,11}$ A split-face comparison study revealed that the higher-energy, lower-density settings of the ablative fractional lasers were more effective for treating atrophic scars and improving skin textures than lower-energy, higher-density settings. ${ }^{12}$ Meanwhile, however, ablative fractional laser treatments at lower-energy, higher-density settings were found to be more effective for dyschromic skin lesions, compared with higher-energy, lowerdensity settings. ${ }^{12}$

Low-fluenced Er:YAG resurfacing generates laserinduced ablative tissue reactions confined to the outer layers of the epidermis for skin rejuvenation or for treating pigmentary disorders. ${ }^{7-9}$ By doing so, laser pulses can be safely delivered to a target area at over 100\% percent coverage while preserving the structural integrity of the basement membrane. ${ }^{7}$ Moreover, low-fluenced Er:YAG resurfacing induces the expression of various cytokines and chemokines for wound repair and tissue remodeling, and also increase the production of type I and type III procollagen in the dermis. ${ }^{7}$ Thereby, clinical improvements in photodamaged skin, which include wrinkles, textural impairments, melasma, and other pigmentary disorders, can be expected after low-fluenced Er:YAG resurfacing 
treatment..$^{7-9}$

Theoretically, the high-energy and low-density settings of ablative fractional lasers seem more profitable for treating dermal scar-like lesions, whereas the lowenergy and high-density settings seem more effective for treating hypopigmented lesions in patients with striae distensae. Accordingly, in this report, we performed ablative, fractional Er:YAG laser treatment at a fluence of $37 \mathrm{~J} / \mathrm{cm}^{2}$ and $11 \%$ percent coverage along the hypopigmented, atrophic lesions. Then, low-fluenced Er:YAG resurfacing treatment was additionally applied around the lesions of striae distensae at a fluence of $2 \mathrm{~J} / \mathrm{cm}^{2}$ and an overlap percentage of $30 \%$.

As stated earlier in this report, one patient with late stage striae distensae experienced postinflammatory hyperpigmentation along the areas that were treated with ablative, fractional Er:YAG laser treatment, not with lowfluenced Er:YAG resurfacing treatment. Nonetheless, her skin lesions showed marked clinical improvement after the single session of combined Er:YAG laser treatment. We suggest that the additional low-fluenced Er:YAG resurfacing treatment could provide synergistic effects on the improvement of atrophic lesions. Meanwhile, the other patient with early stage striae distensae showed near complete improvement thereof within 2 months after the single session of combined treatment without remarkable major side effects. Accordingly, we deduced that early intervention using combined ablative laser systems could improve overall erythematous and atrophic skin lesions.

In conclusion, we demonstrated in this report that two Korean patients with striae distensae on the trunk could be effectively treated with a single session of combined high-fluenced, fractionated Er:YAG laser and low-fluenced, high-coverage, Er:YAG resurfacing laser treatment. The combination of high-energy, low-density and low-energy, high-density ablative laser pulses could have provided synergistic effects on the pathogenetic factors of striae distensae in the epidermal and dermal components. Additionally, we deemed that ablative tissue reactions in the outer layers of the epidermis generated by low-fluenced laser pulses also improve skin tone and texture along the atrophic skin lesions. We believe that combined Er:YAG laser treatments can be effectively and safely used in both early and late stages of striae distensae in Asian patients.

\section{ACKNOWLEDGEMENTS}

We would like to thank Duke Song IConsultant; Sciton,
Palo Alto, CA, USA) for his assistance with technical support. We would also like to thank Anthony Thomas Milliken, ELS at Editing Synthase (https://editingsynthase. coml for his help with the editing of this manuscript.

\section{CONFLICT OF INTEREST}

The authors declare no conflicts of interest.

\section{REFERENCES}

1. Hexsel D, Soirefmann M, Porto MD, Schilling-Souza J, Siega C, Dal'Forno T. Superficial dermabrasion versus topical tretinoin on early striae distensae: a randomized, pilot study. Dermatol Surg 2014:40:537-44.

2. Kim H, Yoo KH, Zheng Z, Cho SB. Pressure- and dosecontrolled transcutaneous pneumatic injection of hypertonic glucose solution for the treatment of atrophic skin disorders. J Cosmet Laser Ther 2017;19:479-84.

3. Kang S. Topical tretinoin therapy for management of early striae. J Am Acad Dermatol 1998;39:S90-2.

4. Lee SE, Kim JH, Lee SJ, Lee JE, Kang JM, Kim YK, et al. Treatment of striae distensae using an ablative 10,600-nm carbon dioxide fractional laser: a retrospective review of 27 participants. Dermatol Surg 2010;36:1683-90.

5. Kim IS, Park KY, Kim BJ, Kim MN, Kim CW, Kim SE. Efficacy of intradermal radiofrequency combined with autologous plateletrich plasma in striae distensae: a pilot study. Int J Dermatol 2012;51:1253-8.

6. Cho SB, Lee SJ, Cho S, Oh SH, Chung WS, Kang JM, et al. Nonablative 1550-nm erbium-glass and ablative 10 600-nm carbon dioxide fractional lasers for acne scars: a randomized split-face study with blinded response evaluation. J Eur Acad Dermatol Venereol 2010;24:921-5.

7. Orringer JS, Rittié L, Hamilton T, Karimipour DJ, Voorhees JJ, Fisher GJ. Intraepidermal erbium:YAG laser resurfacing: impact on the dermal matrix. J Am Acad Dermatol 2011;64:11928.

8. Attwa E, Khater M, Assaf M, Haleem MA. Melasma treatment using an erbium:YAG laser: a clinical, immunohistochemical, and ultrastructural study. Int J Dermatol 2015;54:235-44.

9. El-Domyati M, El-Ammawi TS, Medhat W, Moawad O, Mahoney MG, Uitto J. Multiple minimally invasive Erbium:Yttrium Aluminum Garnet laser mini-peels for skin rejuvenation: an objective assessment. J Cosmet Dermatol 2012;11:122-30.

10. Goldberg DJ, Sarradet D, Hussain M. 308-nm excimer laser treatment of mature hypopigmented striae. Dermatol Surg 2003;29:596-8.

11. Ryu HW, Kim SA, Jung HR, Ryoo YW, Lee KS, Cho JW. Clinical improvement of striae distensae in Korean patients using a 
combination of fractionated microneedle radiofrequency and fractional carbon dioxide laser. Dermatol Surg 2013;39:1452-8.

12. Jung JY, Lee JH, Ryu DJ, Lee SJ, Bang D, Cho SB. Lowerfluence, higher-density versus higher-fluence, lower-density treatment with a 10,600-nm carbon dioxide fractional laser system: a split-face, evaluator-blinded study. Dermatol Surg 2010;36:2022-9. 